\title{
Dynamic Spectrum Management with Network Function Virtualization for UAV Communication
}

\author{
Zhengjia Xu ${ }^{1}$ (D) . Ivan Petrunin ${ }^{1} \cdot$ Antonios Tsourdos $^{1}$
}

Received: 11 June 2020 / Accepted: 6 January 2021 / Published online: 3 February 2021

(C) The Author(s) 2021

\begin{abstract}
Rapid increases in unmanned aerial vehicles (UAVs) applications are attributed to severe spectrum collision issues, especially when UAVs operate in spectrum scarce environments, such as urban areas. Dynamic air-to-ground (A2G) link solutions can mitigate this issue by utilizing programmable communication hardware in the air and real-time assignment of spectrum resources to achieve high-throughput and low-latency connectivity between UAVs and operators. To mitigate the highcomputation issue among ground control station (GCS) networks and provide a broad communication coverage for large number of UAVs, we propose an advanced UAV A2G communication solution integrated with the dynamic spectrum management (DSM) and network function virtualization (NFV) technology to serve urban operations. The edge-cutting UAV communication technologies are surveyed. The proposed scheme is discussed in terms of the high-level system architecture, virtual network architecture, specific virtual functions (SVFs), and affiliated operation support databases. Some major research challenges are highlighted and the possible directions of future research are identified.
\end{abstract}

Keywords Aeronautical cognitive communication · Air-to-ground communication · Virtual network · UAV communication $\cdot$ Network function virtualization $\cdot$ Urban communication

\section{Introduction}

Unmanned aerial vehicles (UAVs) have drawn tremendous attention in recent decades due to their advantages in high mobility and convenient deployment; however, the inherent high-dynamic characteristic [23] in UAVs brings additional challenges in the information exchange between UAVs and users, specifically demanding a low-latency and highthroughput wireless air-to-ground (A2G) link. The communication schemes in $A 2 G$ links typically employ a static link, such as aircraft communications addressing and reporting (ACARS), global system for mobile communication (GSM), and L band digital aeronautical communications systems (L-DACS) [31], with predetermined spectrum resources, which may result in a scarcity of bandwidth in cases of high-throughput communication during short-term

Zhengjia Xu

Zhengjia.Xu@cranfield.ac.uk

1 School of Aerospace, Transport and Manufacturing, Cranfield University, MK43 0AL Wharley End, UK periods [66]. In some operations with extreme spectrum scarcity, such as the urban environment, communication links are easily interfered with by other users, and sufficient bandwidth is lacking. Apart from investigating more efficient links, other considerations are highlighted when operating in the urban area: (1) the strong scalability with a number of UAVs, (2) enabling the deployment of terrestrial infrastructures with broad coverage purpose, (3) mitigating the computation loads, (4) heterogeneous communication technologies, (5) and least modification to the current resolutions. Regarding that most UAV communication researches only work on the device to device solution, those work do not address the above considerations thoroughly, which drives needs of investigating the high-level UAV communication design with edge-cutting technologies.

Several projects has been held to enhance the aeronautical link quality by introducing more flexibility in the communication. In the design phase B of the satellite communication for air traffic management (Iris) project held by the European Space Agency, an asynchronous codedivision multiple-access (A-CDMA)-based return link, i.e., $\mathrm{A} 2 \mathrm{G}$, and an adaptive forward link, i.e., ground-to-air (G2A) synthesized with changeable coding and modulation 
techniques, are being held to maximize the utilization of the aeronautical communication spectrum [30]. The integration of multiple data links into one platform is another challenge to enable the seamless connectivity. SANDRA project held by the German aerospace center (DLR) investigates the integration of data links, radios, and antennas, such as very high frequency data link mode 2 (VDL2), and L-Band and aeronautical mobile airport communication system (AeroMACS) data links. The SANDRA project developed a new aeronautical telecommunication network/ open systems interconnection (ATN/OSI) over VDL2/IP (AeroMACS, SwiftBroadband (SBB)) stack and tested it with flight trials [57].

However, the above projects are designed for aircraft operations without concerning the large UAV numbers and the scarcity of using spectral resources in UAVs. The UAV communication links also demand a simplified resolution with the least modification to the current technologies so as to improve the link flexibility and reliability. Moreover, the scarcity of spectrum resources in UAV operations and lacking the unified UAV communication scheme also drive the need of an integrated communication proposition to alleviate the above issues. The authors in [65] summarized detailed challenges in the design phase of UAV communication solutions where some wireless communication challenges were highlighted, such as the extension of coverage area, the reliable network connectivity and the topology control among UAV networks. The authors in [27] investigated a survey of UAV communication from the link perspective to meet standard requirements of synchronization, safety, security, and scalability, where the feasible wireless technology and adaption of protocols for aerial networks were highlighted.

In an attempt to manage the spectrum resources in the urban area, the resource allocation based dynamic spectrum management (DSM) scheme is promising to introduce more flexibility in the link solutions among UAVs. Enabled with DSM, the spectrum resources can be improved efficiently by way of generating the optimized communication schemes to be accustomed to the changeable RF environment. Some functions are enabled with the DSM, such as the collaborative communication [13], regulated communication behavior [94], and frequency hopping [83]. However, with the centralized DSM schemes [94], the construction of terrestrial infrastructures is one challenge given the temporal and spatial characteristic of spectrum resources for different areas, and high computation when processing and allocating spectrum resources. Moreover, for the urban operation, the integration of heterogeneous infrastructure type is important, which is not considered in most publications.
As a fundamental technology proposed by European telecommunications standards institute (ETSI) in 2012 and used for the $5 \mathrm{G}$ developments, network function virtualization (NFV) technology enables the feasible establishment and integration of infrastructures by decoupling and splitting the entire system functionality of the physical networks into the virtual functions so as to form virtual networks (VNs) [38]. Enabled with the isolated feature of NFV, the hardware deployment issues, such as limitations in the processors, storage, and switches are mitigated from the development perspective. The virtual functions (VFs) are executed on the virtualized platforms simultaneously to leverage the performance and running cost. The software developers program the system functions by requesting the hardware resources, where the hardware resources can be regarded as black boxes. The hardware implementation becomes convenient and the compatibility concern among heterogeneous hardware platforms is addressed by the unified protocol in NFV. Several benefits are highlighted, for instance, having a strong scalability and capability in embracing the heterogeneous hardware, the efficient management of computational resources, reduction in capital expenses, reduction in operating expenses and convenient migration to additional systems. Based on NFV, network slicing, edge computing and software defined network (SDN) are achievable to provide more specific services.

Oriented from the dynamic spectrum sharing structures $[13,83,94]$, this paper proposes to integrate the NFV with the GCSs deployment where the central allocation scheme is employed to serve the UAV operations. The GCSs are formed as VNs. The spectrum resources are processed and managed in VNs. The functions, such as spectrum processing and allocation, and the resource storage in the GCS networks, are also virtualized to facilitate the convenient deployment of GCSs. The contribution of this paper is the integration of DSM and NFV in a general-purpose UAV communication networking to form a unified architecture. Facilitated by the proposed scheme, computation loads in GCSs and UAVs are mitigated. Moreover, the heterogeneous UAV platforms can be embraced conveniently, and other systems are easily interfaced through the application layer directly.

\section{Related Work}

The emergence of cognitive radio (CR) [50] is promising for achieving the flexible communication by using programmable hardware, such as the software-defined radio (SDR). Several parameters are configurable during the transmission in an attempt to maximize spectrum resource utilization and 
maintain quality of service (QoS). Functions of the spectrum resource sharing and dynamic scheduling of communication schemes are commonly considered in the cognitive communication. In CR, two types of users are categorized in accordance with having licenses: primary users (PUs) and secondary users (SUs). SUs are allowed to access channels or frequencies opportunistically when PUs of this channel are idle, where such vacant opportunities are called white spaces, spectrum holes or spectrum opportunities [19, 28].

When applying the CR technology for the UAV applications, extensive works have been done. The authors in [59, 60] discussed typical challenges and solutions when using the CR concept in the UAV communication, for instance the agile link leading to the link losses, distinguished RF environment in different locations, and the UAV mobility leading to propagation effects. The authors in [62] reviewed some design issues, research challenges and promising technologies following the OSI protocol stack for the CRenabled UAV communication. The authors in [30] reviewed CR-enabled aeronautical communication and presented an overview of standards that new systems should be compliant with.

Oriented from the CR concept, the opportunistic spectrum access (OSA) is a more specialized technology aimed at improving the spectrum utilization ratio by sharing spectrum resources opportunistically among users. The authors in [71] presented a multi-level system framework with an opportunistic communication scheme in which the time, frequency and spatial stream of the wireless environment were significantly improved. The authors in [8] described a policy-based radio for UAVs in which the radio can consider spectrum opportunities and perform dynamic spectrum access (DSA). The authors in [67] presented a navigation data-assisted OSA scheme for heterogeneous UAV networks by flexibly scheduling the spectrum subbands. The authors in [45] presented an OSA scheme based on an efficient spectrum sensing algorithm and discussed the determination of spectrum processing time and priority control with the proposed algorithm. However, the above solutions assume to have high-performance processors onboard to implement the real-time spectrum detection [5] (although the size, weight and power (SWaP) limitation can be mitigated through the cooperative sensing [5], such as the work done in [58]), decision-making, and may have the contention issue when UAVs are deployed in a distributed manner.

In addition to providing low-cost methodologies to relieve onboard computational loads, the spectrum resource allocation structure [77] is another resolution to address the limited on-board computation issue. In a wireless sensor network (WSN), a centralized approach was presented to leverage computation for cooperative spectrum sensing with SUs that may use heterogeneous detectors [13]. In aeronautical communications, the authors in [94] presented a framework in which spectrum resources are originally allocated to nodes to ensure resource utilization efficiency. We previously investigated a cognitive-enabled DSM communication scheme for the UAV A2G link where both spectrum awareness and decision making are performed terrestrially and uploaded to the airborne system [83]. However, the above works did not consider the limitation in processing and storing large quantities of spectrum data, especially when monitoring the spectrum over a wide bandwidth.

In recent years, NFV-related research for UAV applications has attracted enormous attentions in many applications. To enable a UAV-assisted information collection network, a softwarization NFV and SDN-based architecture was proposed in [63], where UAVs were combined with WSNs to form a cooperative resource sensing system. A UAV-cloud platform was discussed in [46], where UAVs and services were modeled in a general spectrum allocation based architecture. A UAV system architecture using fog computing capabilities to support Internet of Things (IoT) applications was presented in [51]. To apply NFV in UAV deployment, a UAV scheduling policy was investigated in [76] by having an NFV paradigm to allocate UAVs based on energy consumption. [52] explored and performed preliminary experiments on NFV for enabling the flexible and cost-effective deployment of UAVs with multimission types. The authors in [79] used an NFV-SDN for monitoring UAV-network services, specifically the anomaly detection through SDN traps.

Summaries of the dynamic A2G communication schemes are highlighted in Table 1. The few existing works related to sharing spectrum resources with UAVs, especially with the interweave method, allow UAVs to access spectrum resources opportunistically [66]. Recently, the concept of using the centralized spectrum allocation scheme for the UAV communication is proposed in [9], which is similar with our previous work in [83]. However, both works do not consider the actual deployment of GCSs, as well as huge computation loads in GCSs. Other similar networks, such as VNF- or WNF-enabled radio access network (RANs), are currently being investigated among other application scenarios, for instance, cellular communication in [54] and automotive vehicles in [53]. However, the above works did not consider the typical UAV communication scenario, which drives the needs of proposing an exclusive UAV communication solution for the urban area.

The rest of this paper is organized as follows: Section 3 introduces state-of-art communication networks. Section 4 presents the proposed communication network for UAV 
Table 1 Summarized drawbacks and challenges in the published works

\begin{tabular}{|c|c|c|c|c|c|}
\hline References & Main contribution & Application & $\begin{array}{l}\text { Efficient } \\
\text { spectrum usage }\end{array}$ & $\mathrm{SWaP}$ & $\begin{array}{l}\text { Efficient ground } \\
\text { network }\end{array}$ \\
\hline$[59,60]$ & $\begin{array}{l}\text { Formulate a dynamic scheme } \\
\text { with programmable hardware }\end{array}$ & UAV A2G & $\mathrm{N}$ & $\mathrm{N}$ & N/A \\
\hline$[8,71]$ & $\begin{array}{l}\text { Address spectrum scarcity prob- } \\
\text { lem by opportunistic access to } \\
\text { links }\end{array}$ & UAV A2G & $\mathrm{Y}$ & $\mathrm{N}$ & N/A \\
\hline$[45,67]$ & $\begin{array}{l}\text { Address spectrum scarcity with } \\
\text { OSA considering computational } \\
\text { loads }\end{array}$ & UAV A2G & $\mathrm{Y}$ & $\mathrm{Y}$ & N/A \\
\hline$[13,83,94]$ & $\begin{array}{l}\text { Present spectrum allocation } \\
\text { enabled approaches with } \\
\text { detectors to end users }\end{array}$ & WSN, Aeronautical, UAV A2G & $\mathrm{Y}$ & $\mathrm{Y}$ & $\mathrm{N}$ \\
\hline$[46,51,52,63,76,79]$ & $\begin{array}{l}\text { Present UAV and NFV com- } \\
\text { bined networks among diverse } \\
\text { applications }\end{array}$ & UAV-cloud, FANETs & N/A & $\mathrm{Y}$ & $\mathrm{Y}$ \\
\hline$[38,53,54]$ & $\begin{array}{l}\text { Present communication link solu- } \\
\text { tions with VNs }\end{array}$ & Cellular & $\mathrm{Y}$ & N/A & $\mathrm{Y}$ \\
\hline
\end{tabular}

A2G links. Section 5 highlights research challenges in the implementation of the proposed network. Section 6 concludes this paper.

\section{Architectures of State-of-the-Art Communication Networks}

This section presents the published cognitive communication networks for UAV A2G links, where the cognitive concept is discussed in particular. The typical NFV network in cellular networks is also briefly discussed, given the employment in the proposed network.

\subsection{Cognitive Radio}

In the case of $\mathrm{CR}$, three typical wireless design paradigms are unified according to the coordination relations between PUs and SUs, i.e., interweave paradigm, underlay paradigm, and overlay paradigm [20].

The basic concept of the interweave paradigm is characterized as one opportunistic communication scheme using DSA methods [20], where SUs perform transmission when PUs are vacant. Such vacant opportunity is commonly defined in the frequency and time domain or from the spatial and temporal perspective. One major characteristic of interweave communication diagrams envisions low interference towards existing users, where only the knowledge base of users' patterns is needed and can be identified using spectrum sensing technologies.

The underlay paradigm allows both PUs and SUs to communicate simultaneously only if the interference caused by SUs is below a desired value to PU receivers
[20]. Therefore, the underlay paradigm requires omniscient knowledge of PU locations and relies on a precise propagation model to calculate the SU transmission power beforehand. A thorough system that integrates every user together and manages spectrum among users is necessary in the underlay paradigm, whilst it is not achievable in the industrial scientific medical (ISM) band because users in ISM have not been registered in any systems for now.

The overlay paradigm assumes that SUs are omniscient on PUs' codebooks and messages [20]; thus, SUs perform as transceivers following the procedure of packing $\mathrm{SU}$ messages into the same profile as PUs and broadcast the combined messages to both $\mathrm{SU}$ receivers and $\mathrm{PU}$ receivers.

In practice, the underlay and overlay paradigms require a pre-knowledge or estimation of PUs' information, such as the message contents, transmission power, and receivers' locations; thus, the interweave paradigm is mostly considered especially in DSM.

According to our previous work [83], a general procedure of interweave-based cognitive communication is illustrated in Fig. 1. Two abstract components are defined from the cognitive concept, i.e., cognitive detector (CD) and control agency (CA). The global objective of CDs is to perform spectrum awareness, along with additional functions of sensing a radio frequency (RF) environment, identifying RF patterns, and extracting characteristics in the RF. Some technologies are commonly utilized when constructing CDs, such as the spectrum sensing methods reviewed in [88], identification of spectrum opportunities [14], or utilization of radio environment maps (REMs) [55]. In accordance with the prediction of the PUs' traffic model, two groups of detectors are then categorized, i.e., reactive 
Fig. 1 General procedure of interweave paradigm-based cognitive communication

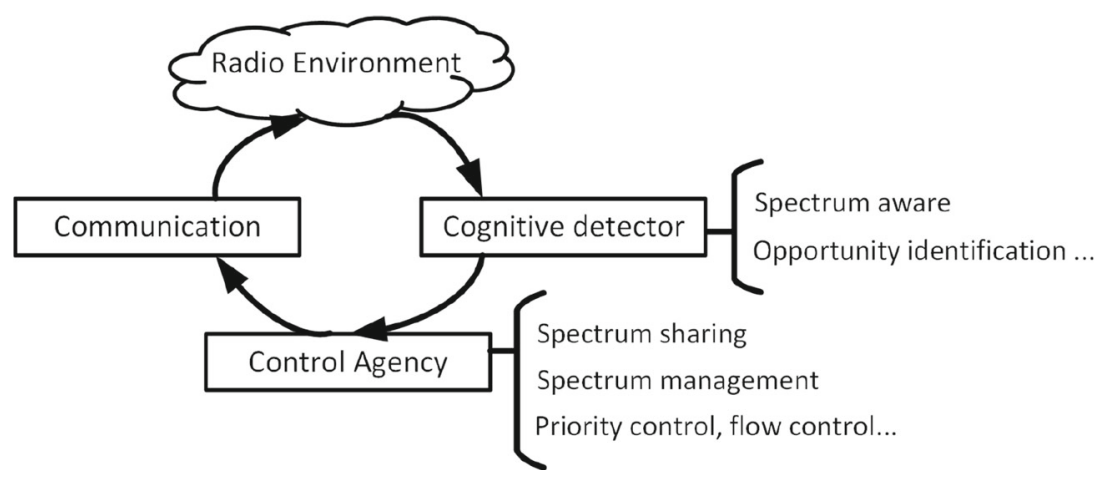

detector and proactive detector [78], based on reactive sensing and proactive sensing approaches, respectively.

The typical CA manages the utilization of spectrum resources from detectors, specifically providing services of intelligent decision making, failure detection and recovery, communication management, priority control, communication load balance among resources.

\subsection{Cognitive Communication for A2G Links}

By applying CR concepts into A2G communication, two typical communication frameworks are highlighted in Fig. 2 referring to [62] considering spectrum sensing deployment. In both presented frameworks, CDs are characterized to monitor the interested spectrum frequency, while UAVs function as a CA to retrieve the spectrum data and share with other UAVs to obtain a global vision [62]. Based on obtaining the entire spectrum environment information, the spectrum utilization schemes are then generated by predicting and analyzing idle frequencies or blank spectrum opportunities.

- In the cluster-based spectrum sensing-based communication framework (see Fig. 2a), CDs are organized into clusters composed of member nodes and cluster heads. CDs of member nodes execute the spectrum awareness function and deliver the spectrum data to CDs of the cluster head, consequently transferring to UAVs for further processing and sharing.

Some distinguished requirements or challenges when implementing this framework are (1) high-throughput upload links attributed to large amounts of spectrum sensing information, (2) high computational processors in CDs and UAVs to execute spectrum sensing and spectrum analysis, respectively, and (3) high-throughput air-to-air links when exchanging spectrum information among UAVs.

- In an attempt to reduce the algorithm complexity and enhance the sensing performance [5], a cooperative spectrum sensing-based communication framework is illustrated in Fig. 2b. The spectrum information is collected collaboratively through $\mathrm{CDs}$ and passed to UAVs via uplinks. Individual CDs broadcast spectrum information without having a specific transmitter link cluster head.

Some distinguished requirements or challenges when implementing this framework are (1) high-throughput upload links attributed to large amounts of spectrum sensing information, (2) high computational processors in UAVs to analyze the spectrum information, and (3) high-throughput air-to-air links when exchanging spectrum information among UAVs.

\subsection{Network Function Virtualization}

Virtualization technology enables the isolation of hardware realization and software applications, where users run an emulated environment on their hardware formulated with multiple virtual machines (VMs) to access physical resources. Specifically, the NFV concept separates NFs from dedicated and heterogeneous hardware appliances consolidated through standard information technology platforms, for instance, servers, switches, and storage [26]. The developers concentrate on developing virtual functions (VFs). The coordination of storage and processing among VFs are addressed in an orchestration to enable an efficient utilization of terrestrial computation resources. A typical NFV framework is illustrated in Fig. 3, where the physical hardware, virtual components, VNFs, operation support system (OSS), and hypervisor are commonly included.

The physical layer consists of computing hardware, storage hardware, and network hardware to form the computation resources, and allow virtual functions to access and manage the resources by mapping the hardware to a virtualization format. The virtualization layer, along with its virtual resources, consists of virtual computing, virtual storage, and VNs abstract hardware resources and provides requested resources to VNFs for execution. The routine, VNs management, and VNs access are controlled by an orchestrator in the hypervisor through this layer. The VNs management aims at controlling NFV life-cycle operations 
Fig. 2 Typical cognitive communications with terrestrial spectrum awareness to enable A2G links

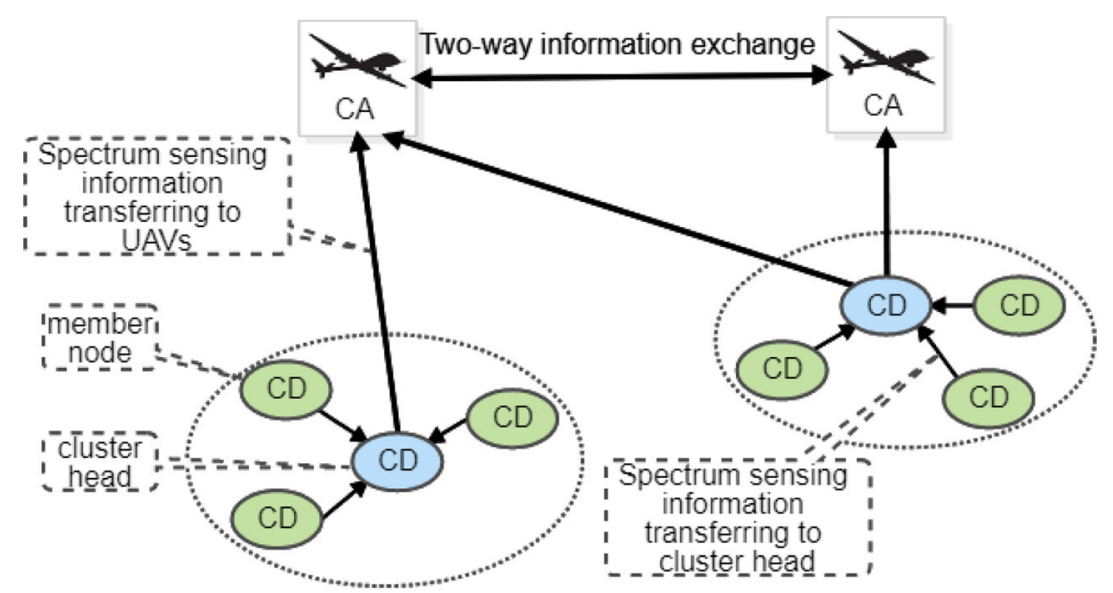

(a) Cognitive communication with cluster-based spectrum sensing.

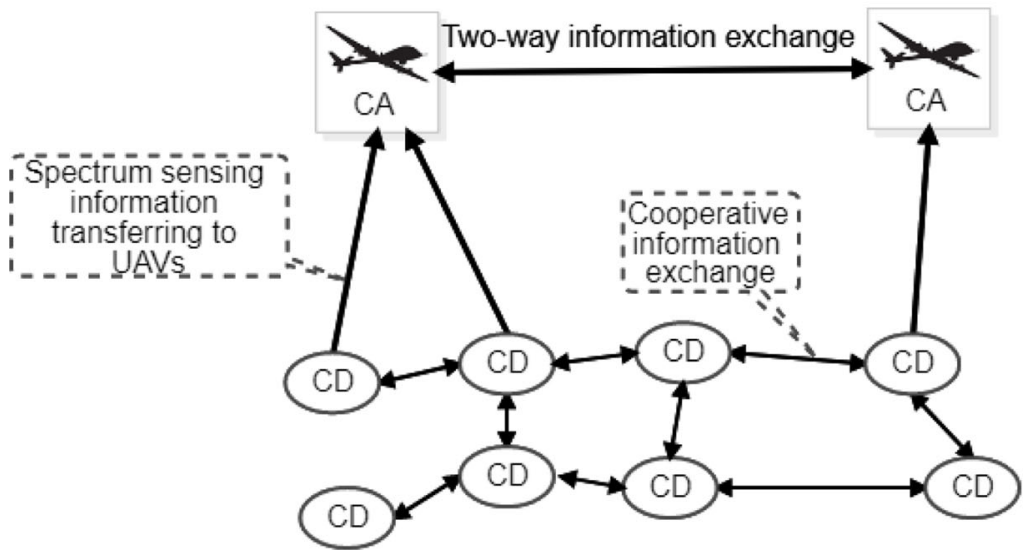

(b) Cognitive communication with cooperative spectrum sensing. and consists of the instantiation, decomposition and deployment [25], update, query, scaling, and termination. Moreover, given the decoupling physical hardware with VNFs, where a virtual infrastructure manager is used for regulating procedures, allocating and managing resources, and detecting or recovering faults in processing.
Fig. 3 NFV framework entities in a cellular network

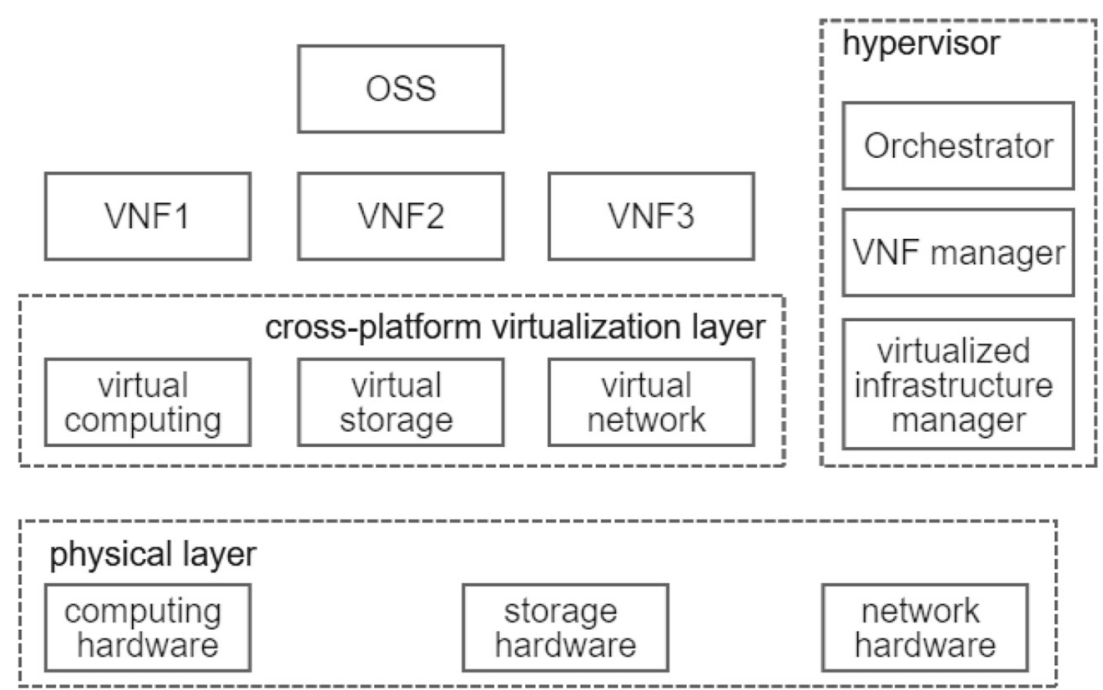


Other components, such as the element management system, service, VNF and infrastructure description, and business support system, are well explained in [26].

Three virtual platforms are categorized according to the service offering levels: software as a service (SaaS), platform as a service (PaaS) and infrastructure as a service (IaaS)) models. With a software customization concept, SaaS offers integrated applications running on VNs to customers via application programming interfaces (APIs). Paas provides the running environment for the customers. Iaas offers the fundamental services in the infrastructures, such as the storage and computation. Some distinct features of SaaS are highlighted, such as release in constructing service users, flexibility and convenience in deployment and professional support [43]. Considering the above remarks, the NFV-enabled SaaS model is preferred for proposing the communication network.

\section{DSM and NFV Enabled A2G Communication}

To alleviate drawbacks in typical cognitive communication (see Fig. 2) and address other challenges in the UAV communication frameworks in Table 1, a novel edge-cutting communication scheme, i.e., a DSM and NFV based communication, is presented in this section. Some discussions are presented, such as the communication procedure, demonstrative use cases, deployment structures of spectrum managers, and affiliate database construction.

The proposed communication is integrated with the cognitive communication concept for the full exploitation of spectrum resource purposes, where CDs are fused to be aware of a global spectral environment. Additionally, a VN is presented and characterized as a CA to process large quantities of spectrum data. The communication schemes are generated in the $\mathrm{VN}$ according to the real-time spectrum environment and are uploaded to UAVs individually. UAVs are equipped with programmable transmitters and broadcast feedback data to CDs through transmitters and terrestrial antennas.

The conceptual urban operation with the proposed DSM and NFV communication is illustrated in Fig. 4. The global spectral environments are analyzed in VNs through infrastructures to discover the vacant spectrum resources. Therefore, the spectrum awareness function in the typical communication systems is offloaded to the terrestrial stations to mitigate the onboard processing. The communication schemes are generated in VNs and forwarded to UAVs through a fixed link, i.e. satellite communication (SatCom) due to its world-coverage capability. The forward link consists of command \& control (C2) messages and the communication scheme. After UAVs retrieve the information, a dynamic backhaul transmission is implemented following the uploaded information with the DSM scheme in Section 3.1.

A specific use case of package delivery with the DSM and NFV solution is presented in Fig. 5. As is shown, when UAV flying across areas with complicated RF environments, corresponding best link schemes (frequency, modulation, transmission power, etc.) are allocated to UAV so as to improve the reliability for the operation. Moreover, the UAV communications are regulated by such scheme. The spectrum scarcity issue in the static links are mitigated through the dynamic shifting of frequency and technology. The centralized spectrum management structure
Fig. 4 The urban operation with the DSM and NFV

communication networks

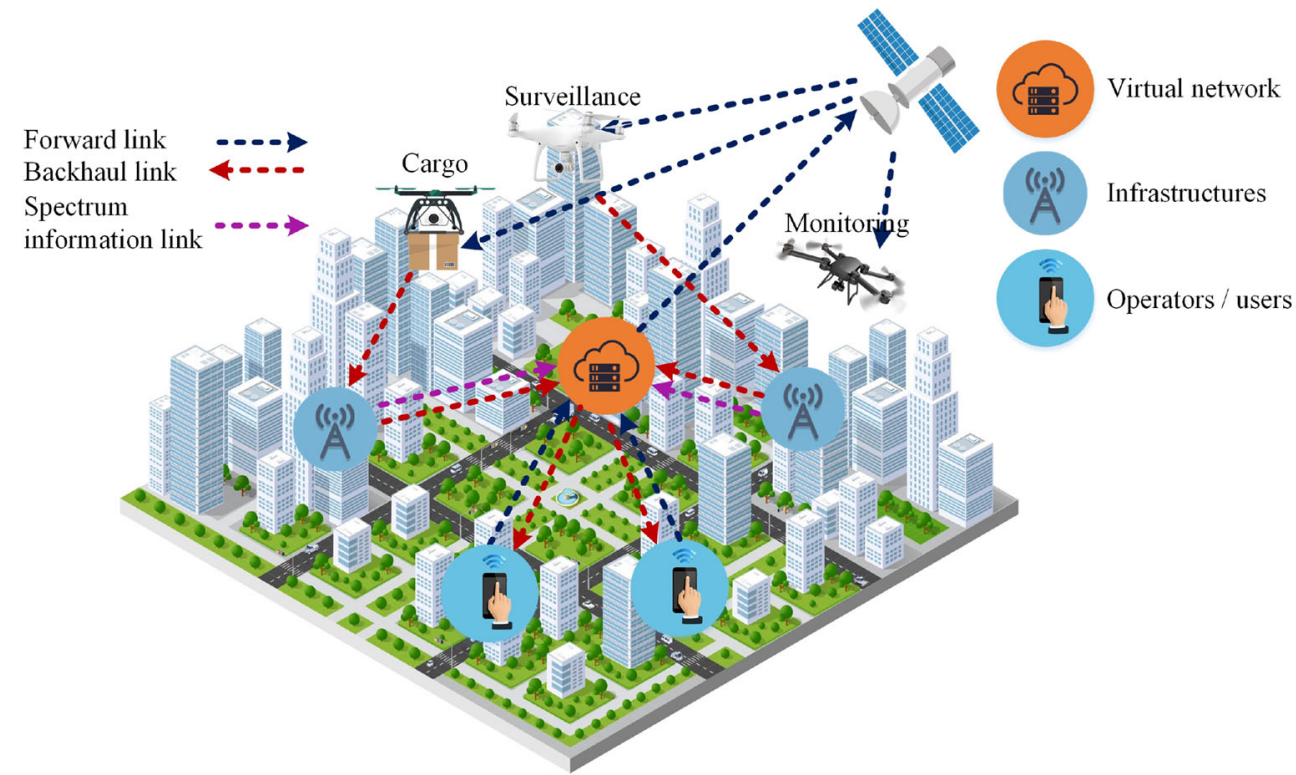


Fig. 5 DSM and NFV based communication for the package delivery

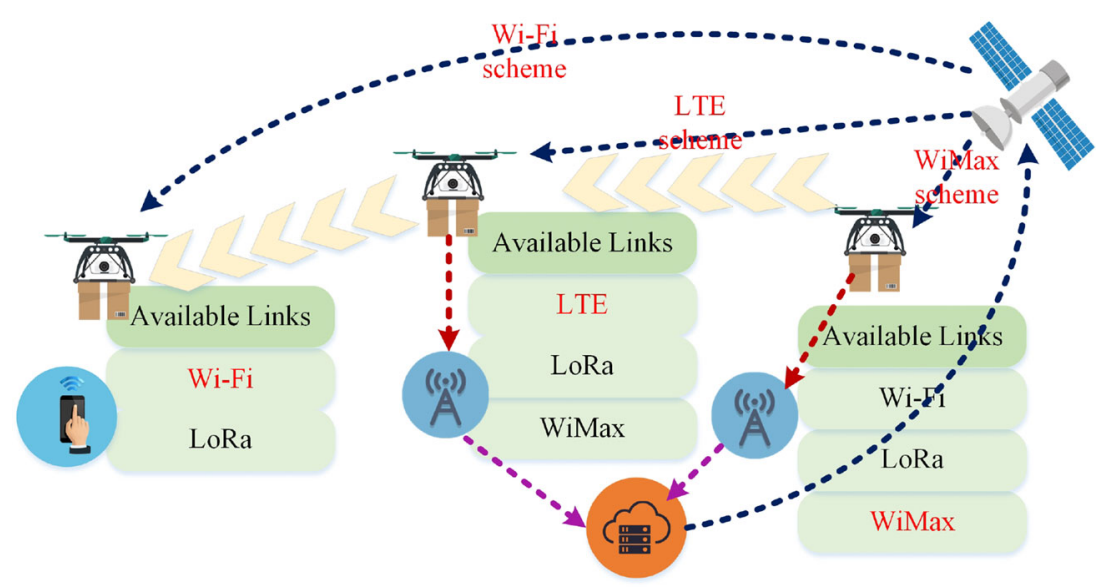

also addresses the issue of RF collision among internal UAV networks.

The proposed DSM and NFV can be developed by modifying current communications. A system model demonstrated with the Internet of Things (IoT) network [3] and the on-board CR communication design [69] is presented in Fig. 6. As the cloud or VNF based IoT has been extensively studied, the terrestrial deployment refers to IoT as the prototype. The sensors are the wideband spectrum sensor in this case, and are enabled with multiple input multiple output (MIMO) technology for detecting $\mathrm{RF}$ environment in interest. The VNs are constructed with typical hardware deployments. The virtual functions are discussed in the following section. The users in this case are the UAV operators, which deliver $\mathrm{C} 2$ messages to VNs, and receive communication messages from VNs.

In a typical UAV control system, some modules are consisted, such as the cognitive communication, flight control, positioning, power supply, sensor fusion, mission execution, and flight management. With the typical CR based UAV design in [69], the cognitive communication module requires to be modified by removing some functions marked in red and adding additional function marked in blue following Fig. 7. Some advanced functions, such as the spectrum selection, spectrum routine, and collision avoidance are mitigated to the $\mathrm{VNs}$ in the proposed architecture. However, the communication scheme, i.e. the spectrum access sequence should be received from GCSs additionally.

The details of constructing VNs with general-purpose conceptions are discussed in the following section.

\subsection{SaaS Deployment}

As is presented in Section 3.3, this paper deploys the SaaS structure as the prototype for interfacing with users and
Fig. 6 IoT and CR based communication model enabled with virtualization technology

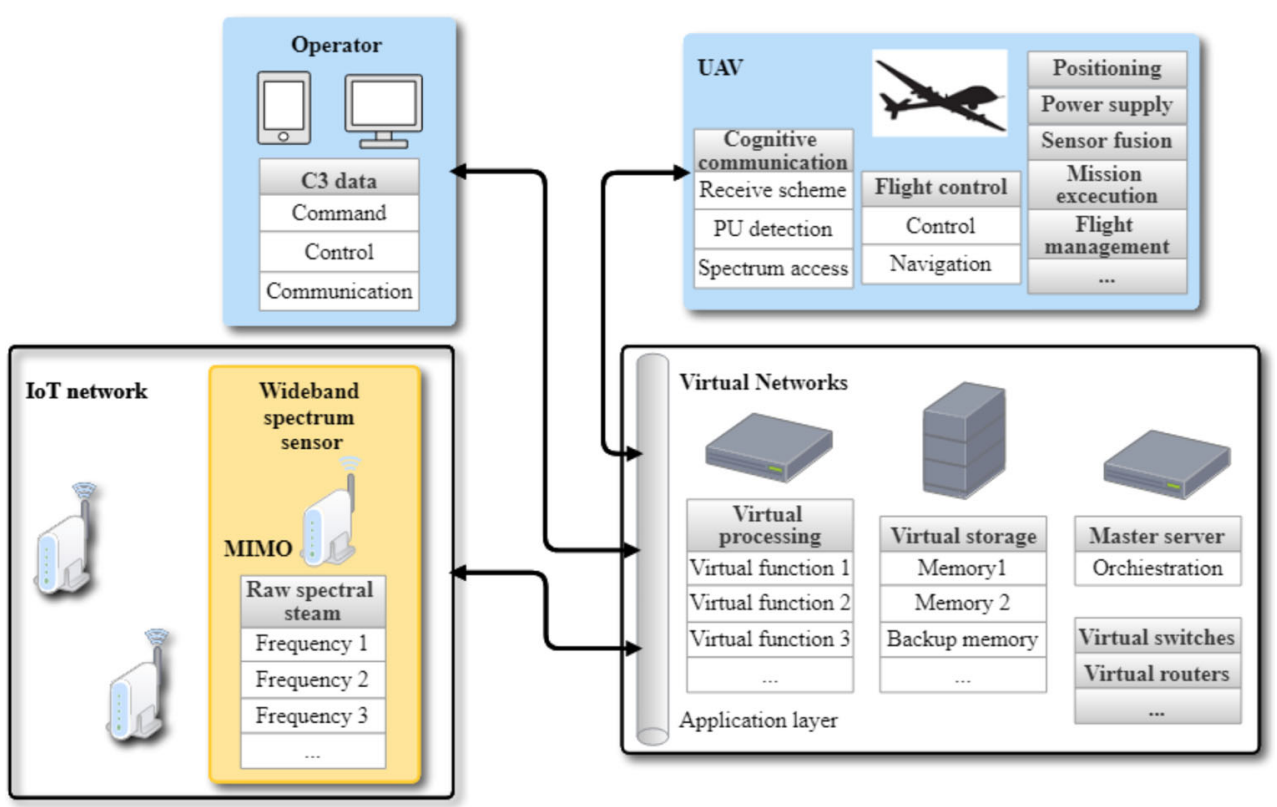


Fig. 7 Hardware deployment with CR architecture. (The offloaded functions are marked as red boxes. The additional function is marked as the blue box)

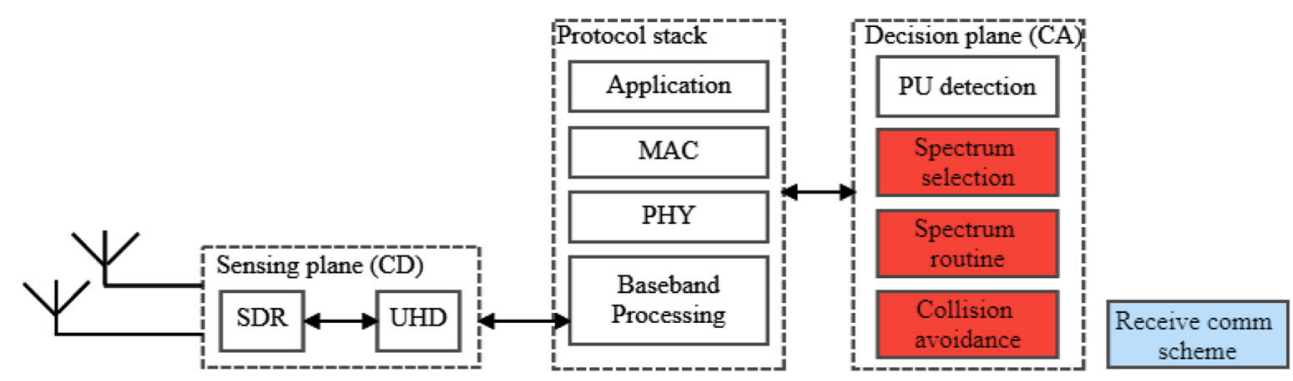

systems. An SaaS based UAV communication structure is illustrated in Fig. 8.

Distinct from the user equipment (UE) in [38], operators in the $\mathrm{VN}$ are defined for the purpose of controlling UAVs and receiving feedback data from UAVs. Specifically, operators send $\mathrm{C} 2$ messages to the $\mathrm{VN}$ and retrieve feedback data from the network.

Similar to the remote radio head (RRH) or remote radio unit (RRU) in [38], cognitive transmitters (CTs) occupy the $\mathrm{VN}$ for accessing the assigned outcomes, i.e., the generated communication schemes. CTs also enable a large number of antennas in their coverage area and operate as transmitters for delivering messages to UAVs via fixed uplink solutions.

Facilitated by the VN, the computation loads among CDs are also released where the main function is to retrieve raw spectrum data over a broadband frequency from a number of antennas. After handling the data briefly, for instance, downsampling, filtering, packing or demodulation, the processed data are then passed to $\mathrm{VN}$ to form a global picture of spectrum environment. It is noted that the sensed data not merely include the information of the RF environment but may also contain the feedback information from UAVs.
Providing that heterogeneous $\mathrm{CD}$ types may exist in practice, the level of heterogeneity is typically accommodated in cooperative spectrum sensing schemes [5] to enable the coexistence of various SU networks. Facilitated with NFV, the handling with heterogeneity can be achieved in the VFs with sufficient computation resources and executed in a central way to obtain an accurate decision. Typical cooperative sensing methods [5] can also be implemented as VFs.

Some other units composed in the VN are highlighted; for instance, a switch [26] is required for message exchange between the $\mathrm{VN}$ and other units, and integration of baseband units (BBUs) is required to realize defined functions, and BBU pool units are built up as local cluster heads in Fig. 2a to manage computational loads and storage based on the global spectrum environment. The connectivity between BBUs and CDs can be fully or partially decentralized to reduce the burden on the transport networks [24].

From the high-level system perspective, the most distinctive feature with typical NFV architectures, such as the one presented in Fig. 3 or the architectures in cellular communications [24], is the changeable network end-users according to the workflow direction.

In the forward workflow, i.e., delivering control messages to UAVs, CTs are end-users of the VN due to the
Fig. 8 Virtualized system architecture for spectrum resource management in UAV A2G communication

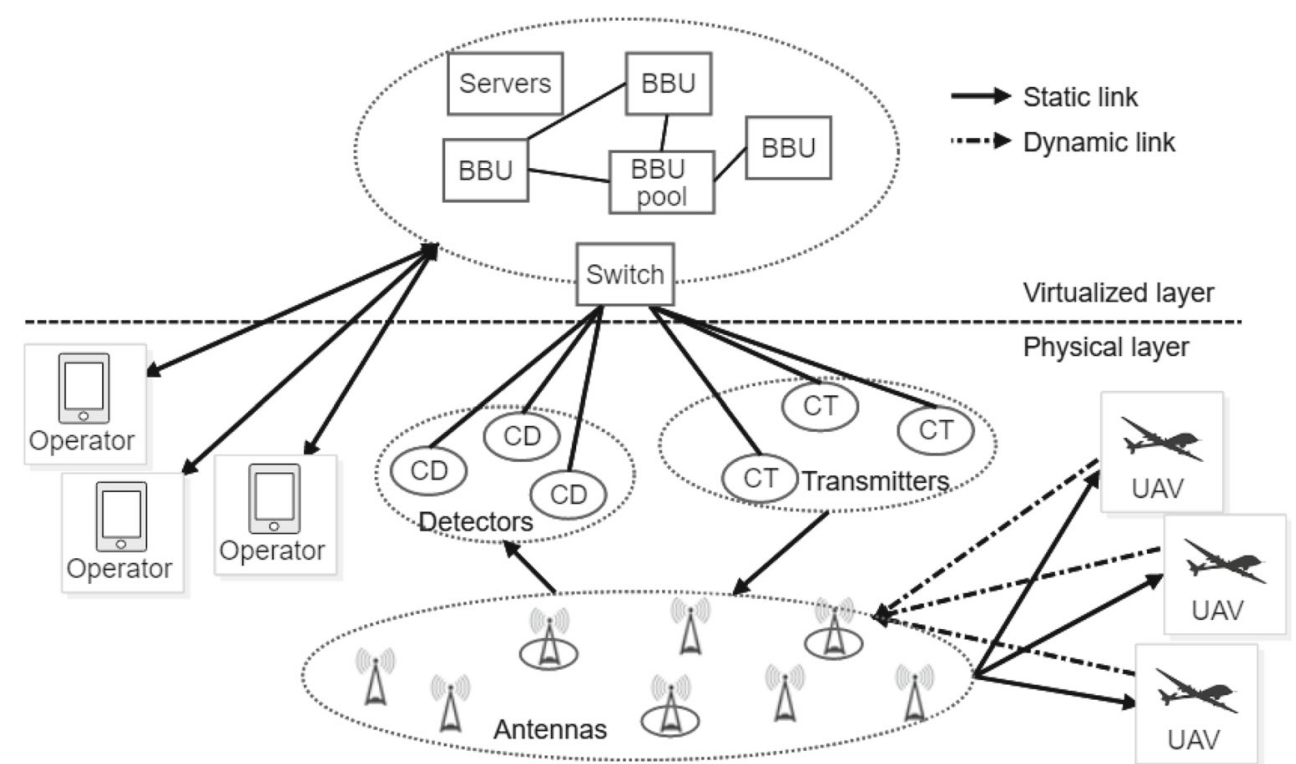


acquisition of the assigned spectrum outcomes. In the backward workflow, i.e., retrieving feedback massages from UAVs, operators become end-users due to the acquisition of information via the network.

\subsection{Virtual Network Architecture}

A fundamental SaaS-enabled VN architecture is highlighted in Fig. 9, where the architecture is organized with a flexible infrastructure layer, support platform, basic applications layer, and applications layer. Three VN utilities, i.e., operators, CDs and CAs, demonstrate access to the proposed network via web services, providing efficient bandwidth of Internet access with web browsers. Distinct from typical architectures such as the SDN-based WNV framework in $[38,53]$, some customized applications are marked as 'green' to distinguish differences.

A number of resources are virtualized and combined through a support platform layer, where physical resources commonly include processors to supply computational power, servers for storage and middleware to provide other functions, e.g., firewalls [79]. Other software resources are orchestrated by this support platform, where the resources include the operating systems (OSs) and network constructions. Facilitated by the additional virtualization plane, the above resources are isolated from the hardware and become flexible to fulfill unique customizations.

A basic application layer is required to formulate the fundamental functions of the VN, e.g., operation and maintenance, access control, and security control.

- Operation and maintenance applications generally monitor the utilization conditions among VMs and balance computational resources to confront an optimal condition in operations. Specifically, the optimization, scheduling [89], dispatching, service oriented, and knowledge management functions are typically considered in resource allocation, as well as fault detection, load balancing [49], state estimation, and high availability in the monitoring phase [87].

- Access control provides admission to certain activities or end-users relying on predefined permissions and privileges. The access control application in this work generally regulates the access behavior from operators to the requested data, while the access among internal activities, such as the connectivity between processor and storage, is also included in this application. Three categories of access control models are typically grouped according to whom owns the authorization: discretionary access control (DAC), mandatory access control (MAC), and role-based access control (RBAC) models [47].

- Security is always one challenge in the VN, providing its high dependence on software engineering and remote access to physical infrastructures. Some secure aspects are summarized, such as data security, abuse of cloud services, malicious insider and cyberattacks [85]. There are a number of solutions to improve network security, such as the identification of access methods, reliable access control, modern virtual private networks (VPNs), and authentication credentials (ACs). A thorough review of cloud service security is discussed in [68].

On the basis of the introduced applications, the highlevel applications are customized according to our particular needs of UAV services, and the fundamental applications are highlighted, such as registration and authorization, web server hosting, and specific virtual functions (SVFs). The

Fig. 9 Network architecture for the SaaS platform

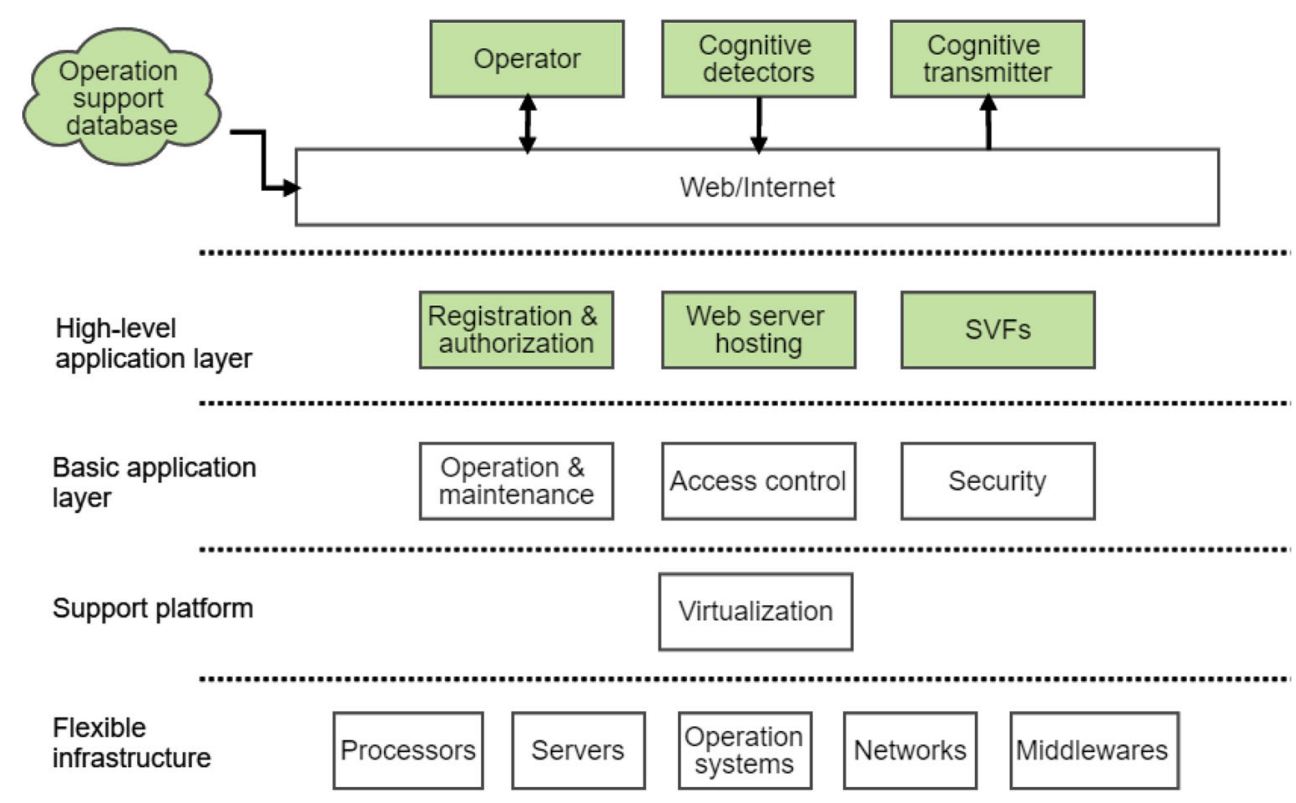


former two applications perform similar functions as the user authentication and web interfaces in [38], while the SVFs are discussed in the following section.

- The registration and authorization application serves to ensure confidential access of operators, CTs and CDs to the VN without security threats. Providing multiple potential end-users, its corresponding utility in a multitenant SaaS platform can be referred to, which allows multiple organizations to be served by accommodating their unique requirements through configuration at the same time [2].

- Distinct from non-VNF enabled web hosting models, the web server hosting in the VN represents that the acquisition of data is performed over an abstract server, even though the data are stored in heterogeneous servers. Therefore, a high-level controller is required for creating web hosting services, balancing services and assigning web server hosting to customers.

\subsection{Specific Virtual Functions}

In this section, we focus on the detailed discussion on the SVFs to meet the minimum construction of the network considering unique UAV features. Furthermore, the forward workflow chart and backward workflow chart based on the VFs are presented in Fig. 10 to explain logical relationships among VFs.

The resource process and storage function aim at retrieving the spectrum data from CDs. Apart from the storage objective, the collected spectrum resources demand to be identified to obtain spectrum opportunities and packed with labels such as time label [61] and geographic information label [18] for the further allocation. Considering the data pass between the process and storage, an efficient pass-byreference data flow scheme then can be referred to [16] because of the virtual storage feature in $\mathrm{VN}$ and main advantages of low throughput and latency in transferring large quantities of data.

In the proposed architecture, the operators communicate with UAVs through the VN, thus a command process and storage function is needed to establish the connectivity, specifically to provide APIs [26] to operators and save C2 messages from operators. Those C2 messages control the high-level operations and are deterministic with some examples of information requests, upload waypoints, operation at a certain condition, return home, etc.

For the purpose of real-time assignment of spectrum resources [77] and mitigation of onboard computational loads, the communication scheduling function is virtualized as one primary service to generate dynamic communication schemes. Embedded with programmable transmitters in UAVs such as lightweight SDR [17], the recommended transmitter configurations, such as modulation type, tone number, and communication frequency, can be optimized according to the collected spectrum environment and then packed into headers with the requested C2 data. By implementing a time-synchronized protocol such as the IEEE 802.1ASrev [10] standard in the UAV platform, the communication scheme labeled with time information, specifically the start time of transmission and the end time of transmission, can also be integrated into the data package as gate control lists (GCLs) [18] for implementing an opportunistic communication solution. Additionally, with sufficient computational power provided by the VN, more considerations can be introduced for producing the communication schemes, for instance, the heterogeneous QoS requirements among various links [84], communication delay compensation based on real-time flight conditions [44], link enhancement and recovery mechanism [73].

A message extraction is prominent for discovering UAV signals in broad bandwidths and decoding feedback data from received signals. Assisted by the knowledge of communication schemes, the extraction can be addressed with UAV estimation of arrival messages. Providing various data types, for instance, the detector data (e.g., messages, images, video, etc.), health data (e.g., remaining power, mechanical/electrical failure) or other specific types [83], it is possible to receive multiple data segments when the communication scheme is offered with short duration of spectrum opportunities. Consequently, the functionality of combining multiple data segments into one is formulated [21].

The flight-condition monitoring function facilitates the alleviation of side effects [23] resulted from mobile and dynamic conditions of UAV operation scenarios. Some propagation effects, such as the Doppler effect, multipath effect, and fading effect can be compensated in the modulation assisted with this function. In addition, nontypical flight conditions may also influence communication performance [31], e.g., low remaining power and abnormal altitude, which can be compensated for or handled with on the ground.

The mobile characteristic and flexible data links may introduce more risks, such as loss of connection in the uplink, loss of connection in the feedback link, incomplete data segments, and high package error ratio, comparing with static link solutions. Hence, a function of failure discovery and recovery is needed to maintain the link quality and enable the integrity of feedback data. Moreover, corresponding failure recovery measurements $[44,73]$ are executed in this function to mitigate the discussed negative effects.

It worth to be mentioned that assisted with VNF, the VN can be easily extended with more applications or integrated with other systems, such as network topology maintenance among groups of CDs, CTs and BBUs [1], communication scheduling, coordination and cooperation 
Fig. 10 Architecture and workflow in the $\mathrm{VN}$

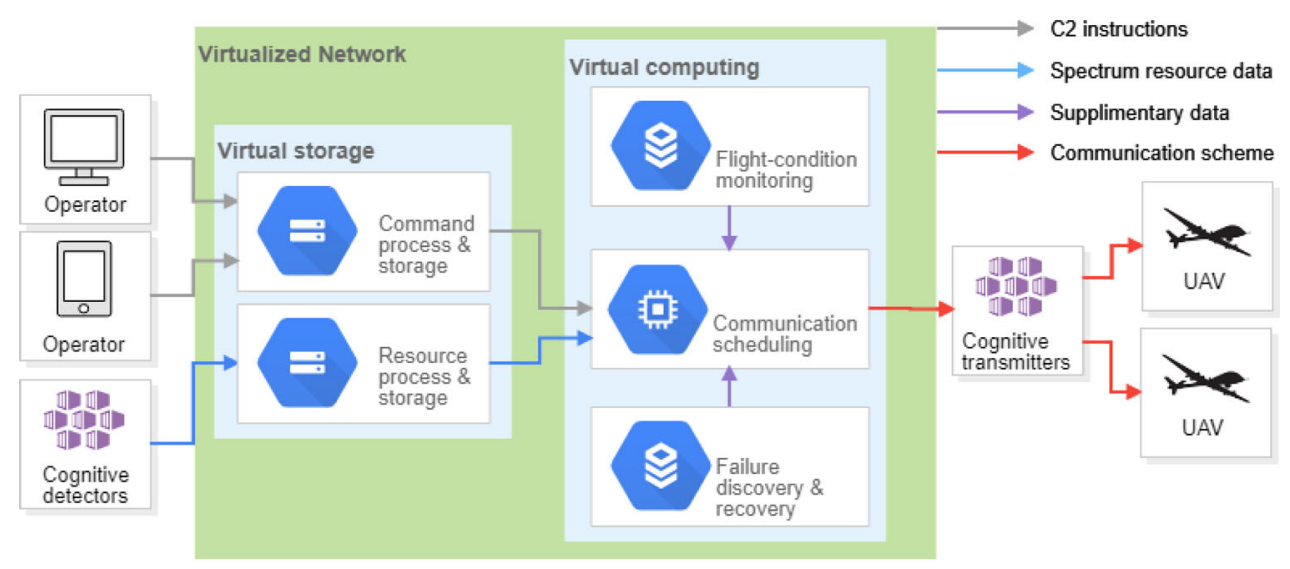

(a) Forward workflow chart.

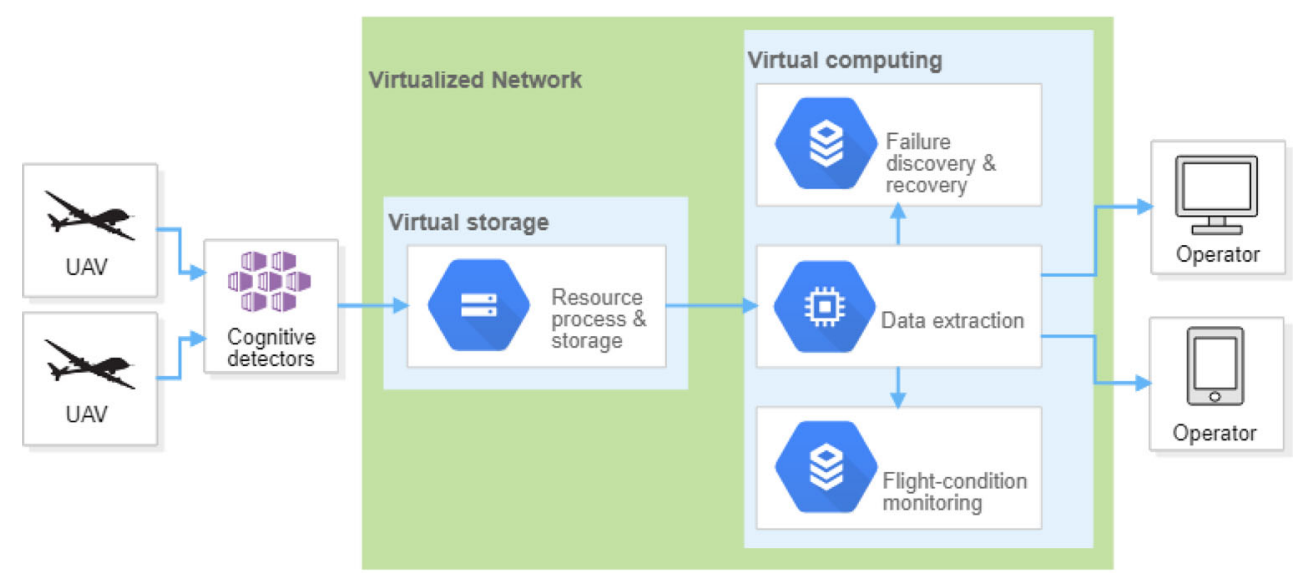

(b) Backward workflow chart.

\subsection{Operation Support Database}

[72], energy spectrum allocation based task allocation [46], and anomaly detection system to maintain network operations [79].

It is noted that facilitated by the operation and maintenance function discussed in the basic application layer, the computational resources among the VFs can be dynamically adjusted to optimal conditions according to the computational status and demands among VFs. For example, when operators send commands to the network, the communication scheduling function is assigned with more computational resources in order to gather information from other functions and generate communication schemes for multiple UAVs. In the idle condition, which means no orders to be given, more computational resources are assigned to the spectrum process function to analyze and estimate spectrum patterns. Similarly, the failure discovery and recovery function, flight-condition monitoring function, and spectrum process and storage function request for computational power only when receiving information from UAVs. Therefore, by having such VFs and dynamic management, the NFs are well assembled and operate in optimal conditions.
Providing the existence of UAS traffic management (UTM) systems [52] or other mature systems, some information can be obtained directly from different database. For the implementation of the $\mathrm{VN}$, some information are needed, such as the UAV location, operational conditions of antennas and preregistered UAV information, thus the UAV geographic database, antenna information database and UAV mission database are highlighted in this section separately.

UAV geographic database is used for recording and updating the location of individual UAVs, where the discovery of UAVs commonly follows an active or passive way. The active discovery method represents UAVs broadcasting locations to ground stations periodically, especially by using automatic dependent surveillance-broadcast (ADS-B) messages. The passive method indicates that UAVs are monitored with additional methods, such as acoustic signature identification [7], optics identification [42], radar-based detection [12], and radio frequency (RF) signal detection [17]. 
Antenna information database matters especially in the heterogeneous networks (HetNets) and beyond visual lineof-sight (BVLoS) operations, providing the storage of antenna deployment [80] and running conditions. This database stores the available antennas and enables the connectivity from the physical layer perspective and also relates to the coverage map [80] of services for supporting UAV operations in this case.

Similar to the airline flight plans [6], the UAV mission database registers flight plans before each operation, including operation number, ID of UAV, start time, end time, destination, start location, operation type, estimated flight path, speed, and altitude [33]. The UAV geographic database and mission database are fused together to enable applications, e.g., scheduling the flight path to maintain an optimal communication connectivity [90], and reducing negative side effects as explained in the failure discovery and recovery function.

\section{Open Issues or Future Directions}

According to the proposed DSM and NFV communication, some research challenges and promising directions are highlighted in this section.

\subsection{Intelligent Spectrum Awareness and Analysis Technology}

The proposed system architecture relies on two critical functions of spectrum awareness: the recognition of the spectrum environment and the identification of the UAV feedback signal. The cognitive receiver specifically enabled with heterogeneous antennas in wideband sensing frequency is commonly recognized to be challenging for simultaneously processing the explosive growth of spectrum data with multiple data types.

To improve cognition in detectors, facilitated by the powerful decision-making capability in deep learning, the integration of deep learning into conventional spectrum awareness methods can be prominent in enhancing the intelligence in any detectors or receivers, as well as improving the end-to-end capability for the reduction in human intervention. Some studies have been performed in the identification of signals by using deep learning approaches. For instance, one study [36] presented an approach for extracting features in the frequency and time domains for blind detection based on the fusion of convolutional neural networks (CNNs) and long short-term memory (LSTM) networks. In [86], a CNN-based module was used to extract Morse signals in a blind wideband sensing scenario. Nevertheless, there are some gaps between current solutions to form integrated and intelligent detectors.

\subsection{Edge Computing Enabled A2G Communication}

In an attempt to release the growth of traffic data and balance cloud computing and edge devices, edge computing or multiaccess edge computing (MEC) paradigms are a promising approach for the near future and can offer an ultra-low-latency environment with high bandwidth [24].

Currently, most studies on edge computing in UAV applications are investigating UAV-assisted mobile communication networks. For instance, the authors in [32] presented a UAV-aided cloud computing system, where UAVs are endowed with computing capabilities to offer computation offloading opportunities to mobile end users. The authors in [11] discussed an edge computing empowered radio access network (EC-RAN), where UAVs are mounted with freespace optical (FSO) in fronthaul and backhaul (FnB) links to assist terrestrial wireless communication. To this end, a specific edge computing assisted UAV communication network is demanded due to the closer distance between utilities and benefits of high-throughput and low-latency services for the UAV A2G links.

\subsection{Efficient Resource Allocation Strategy}

Two use cases of resource allocation strategy exist in the proposed system architecture, i.e., resource allocation in assigning spectrum data and resource allocation in assigning computation among the VNs.

Spectrum resource allocation functions determine the best portions of the spectrum for occupation in accordance with sufficient spectrum resources, where some typical algorithms are graph theory, linear programming, fuzzy logic, game theory and evolutionary algorithms [77]. Given the time-varying feature in spectrum resources, along with its large data size, the algorithm developed with a distributed deployment then becomes meaningful, for instance, an auction-based theory [29], game theory [15] and local bargaining approach [93]. Additionally, the context-aware allocation solution [40] is achievable by having such a spectrum omniscient network.

Similarly, the resource allocation in VNs is also an open issue for optimizing network efficiency, where a number of state-of-the-art methods have been developed, such as a computation energy-aware resource allocation method aiming for managing scientific workflow executions [82], a game-theory-based allocation method [56], a cooperative communication and computational resource allocation algorithm [48], and a service level agreement-based resource provisioning algorithm in [81]. 


\subsection{Vertical Handover and Time Sensitive Networks}

The typical vertical handover or handoff solution controls access to HetNets under the always best connected (ABC) concept following general stages of handover decision, radio link transfer, and channel assignment [4]. The handover decision relates to the selection of access time and the target point of attachment. Radio link transfer is responsible for the formation of a link to the new point of attachment. Channel assignment controls the allocation of channel resources [84].

In the proposed DSM and NFV based system architecture, the high-level resource allocation policy is performed in the cloud, while the low-level execution procedure to access the network is critical, specifically the selection of proper switching time and policy while maintaining the current QoS among links; thus, the vertical handover decision can be referred to. Some typical decision strategies are the decision function (DF), user-centric (UC), multiple attribute decision (MAD), and fuzzy logic and neural network (FL/NN)-based strategies [35]. Moreover, some advanced control methods can be applied as the vertical handover solutions if the PU traffic pattern can be modeled, such as the event-triggered control strategy in [34], the faulttolerant nonlinear fuzzy control method in [74], and neural network based advanced fuzzy control method considering the input saturation in [75].

Given two typical time-triggered (TT) networks of TT Ethernet and time-sensitive networks (TSNs), most papers investigate the scheduling policy [91], update mechanism [41] and performance analysis [92] to eliminate side effects of end-to-end latency and low jitter. However, the above studies are mostly designed in wire connections for industrial applications. For studies on TT wireless communication, TT Ethernet along with IPv6 and lightweight transmission control protocol/ Internet protocol (TCP/IP) is well tested for spacecraft [22], providing the advantages in the deterministic traffic model. The authors in [61] employed a TT hybrid access scheme by using time-division multipleaccess (TDMA) as the prototype based on a time-slot mechanism. Therefore, a time-triggered access solution specifically designed for UAV applications is also prominent for accessing HetNet.

\subsection{Software-Defined Radio Enabled Programmable Communication}

The hardware solution in a cognitive communication system usually utilizes SDR that effectively addresses challenges in parameter configuration in the presence of high variability in the RF environment. SDR also provides the necessary functionality for forming CDs that enable dynamic adjustment of communication parameters, such as frequency, modulation type, and type of signal waveform, maintaining quality of service $(\mathrm{QoS})$ or quality of experience $(\mathrm{QoE})$. SDR also supports effective cross-layer design to optimize communication efficiency and the implementation of dynamic handoff between different modulation schemes or communication technologies, such as global systems for mobile communications (GSM), orthogonal frequency division multiplexing (OFDM), minimum shift keying (MSK), phase-shift keying (PSK), continuous waves (CW), Wi$\mathrm{Fi}, 3 \mathrm{G} / 4 \mathrm{G} / 5 \mathrm{G}, 802.11 \mathrm{~b} / \mathrm{g}$, and chirps [37]. CR-enabled spectrum hopping technology to switch among various frequency bands, such as high frequency (HF), very high frequency (VHF), L-band, S-band, Ku-band, and Ka-band [23], is also achievable with SDR solutions.

\subsection{Radio Environment Map}

Spectrum awareness in REMs has also recently attracted considerable attention. Specifically, the construction, utilization and recognition of REM are the main topics. Taking advantage of REM, the system architecture can be further simplified without consideration of the terrestrial detectors or antennas. The future system can access to REMs, where REMs can be constructed by telecommunication service providers directly.

Some studies related to REM are highlighted, where most studies aim at addressing the construction of REM and the utilization of REM. The authors in [70] presented the construction of a secure REM database for spectrum sharing purposes. A kriging interpolation method was presented in [64] for improving accuracy in acquiring REM data where REM stores the spatial distribution of the average received signal power. The authors in [39] investigated one application of REM for dynamic broadband access in TV bands in urban areas.

\section{Conclusion}

With the explosive growth of operator and UAV numbers, the voluminous data, and requirements for onboard SWaP, efficient network construction and optimal utilization of spectrum resources, UAV A2G claims a deep rethinking of communication network structures, specifically spectrum allocation based communication. Driven by the above motivations, we first reviewed state-of-art UAV communications from typical cognitive communication to OSA and spectrum allocation-based architectures. Typical enabled techniques such as CR and NFV networks were presented, along with CR-based A2G networks. Moreover, we proposed a novel communication network for UAV operations based on DSM and NFV. The high-level system network was modeled with SaaS; the detailed construction of VN was discussed; and 
SVFs were designed to support the unique UAV applications, and the affiliate demanded database was provided. Finally, the open issues were highlighted in the field of implementing such spectrum-oriented UAV communication networks, and future research directions were identified.

The proposed communication enhanced with NFV functionality and DSM concept enables high efficient communication and networking between UAVs and GCSs. Some benefits are highlighted, such as the mitigation of computation and storage constraints among GCSs, lowering operating expenses for UAVs and the infrastructure networks, enabling efficient deployment of GCS networks, reducing risks of communication collisions among UAVs, and more convenient interface to other systems.

Acknowledgements We thank Dr. Runqi Chai for assistance with proofreading and editing. We thank reviewers and editors for comments that greatly improved the manuscript.

Author contributions All authors contributed to the study conception and design. Material preparation, manuscript writing and revision were done by Zhengjia Xu. Review and commentary were done by Dr. Ivan Petrunin. The project administration and supervision were contributed by Prof. Antonios Tsourdos. All authors read and approved the final manuscript.

Funding The authors have no relevant financial or non-financial interests to disclose.

\section{Declarations}

Conflict of interests The authors have no conflicts of interest to declare that are relevant to the content of this article.

Open Access This article is licensed under a Creative Commons Attribution 4.0 International License, which permits use, sharing, adaptation, distribution and reproduction in any medium or format, as long as you give appropriate credit to the original author(s) and the source, provide a link to the Creative Commons licence, and indicate if changes were made. The images or other third party material in this article are included in the article's Creative Commons licence, unless indicated otherwise in a credit line to the material. If material is not included in the article's Creative Commons licence and your intended use is not permitted by statutory regulation or exceeds the permitted use, you will need to obtain permission directly from the copyright holder. To view a copy of this licence, visit http:// creativecommonshorg/licenses/by/4.0/.

\section{References}

1. Abu-Amara, H.: A fast topology maintenance algorithm for highbandwidth networks. IEEE/ACM Trans. Netw. 1(3), 386-394 (1993)

2. Aghera, P., Chaudhary, S., Kumar, V.: An approach to build multi-tenant saas application with monitoring and sla. In: 2012 International Conference on Communication Systems and Network Technologies, pp 658-661 (2012)
3. Ahmed, T., Alleg, A., Marie-Magdelaine, N.: An architecture framework for virtualization of iot network. In: 2019 IEEE Conference on Network Softwarization (NetSoft), pp 183-187 (2019). https://doi.org/10.1109/NETSOFT.2019.8806650

4. Akyildiz, I.F., McNair, J., Ho, J.S.M., Uzunalioglu, H., Wenye, W.: Mobility management in next-generation wireless systems. Proc. IEEE 87(8), 1347-1384 (1999)

5. Althunibat, S., Di Renzo, M., Granelli, F.: Towards energyefficient cooperative spectrum sensing for cognitive radio networks: An overview. Telecommun. Syst. 59(1), 77-91 (2015)

6. Altus, S.: Effective flight plans can help airlines economize. AERO Magazine QTR-03.09(5), 27-30 (2009)

7. Bernardini, A., Mangiatordi, F., Pallotti, E., Capodiferro, L.: Drone detection by acoustic signature identification. Electron. Imaging 2017(10), 60-64 (2017)

8. Brown, T.X., Jaroonvanichkul, S.: Policy-based radios for UAS operations. In: 2012 IEEE Globecom Workshops. IEEE, pp 1614 1619 (2012)

9. Chriki, A., Touati, H., Snoussi, H., Kamoun, F.: Centralized cognitive radio based frequency allocation for UAVs communication. In: 2019 15th International Wireless Communications Mobile Computing Conference (IWCMC), pp 1674-1679 (2019). https://doi.org/10.1109/IWCMC.2019.8766481

10. Craciunas, S.S., Oliver, R.S., AG, T.: An overview of scheduling mechanisms for time-sensitive networks. In: Proceedings of the Real-time summer school LÉ,cole dÉté Temps Réel (ETR), pp 1551-3203 (2017)

11. Dong, Y., Hassan, M.Z., Cheng, J., Hossain, M.J., Leung, V.C.M.: An edge computing empowered radio access network with UAV-mounted FSO fronthaul and backhaul: Key challenges and approaches. IEEE Wirel. Commun. 25(3), 154-160 (2018)

12. Drozdowicz, J., Wielgo, M., Samczynski, P., Kulpa, K., Krzonkalla, J., Mordzonek, M., Bryl, M., Jakielaszek, Z.: 35 GHZ FMCW drone detection system. In: 2016 17th International Radar Symposium (IRS). IEEE, pp 1-4 (2016)

13. Ejaz, W., Hattab, G., Cherif, N., Ibnkahla, M., Abdelkefi, F., Siala, M.: Cooperative spectrum sensing with heterogeneous devices: Hard combining versus soft combining. IEEE Syst. J. 12(1), 981-992 (2018)

14. Elzanati, A.M., Abdelkader, M.F., Seddik, K.G., Ghuniem, A.M.: Adaptive spectrum hole detection using sequential compressive sensing. In: 2014 International Wireless Communications and Mobile Computing Conference (IWCMC), pp 1081-1086 (2014). https://doi.org/10.1109/IWCMC.2014.6906505

15. Etkin, R., Parekh, A., Tse, D.: Spectrum sharing for unlicensed bands. IEEE J. Sel. Areas Commun. 25(3), 517-528 (2007)

16. Friis-Christensen, A., Lucchi, R., Lutz, M., Ostländer, N.: Service chaining architectures for applications implementing distributed geographic information processing. Int. J. Geogr. Inf. Sci. 23(5), 561-580 (2009)

17. Fu, H., Abeywickrama, S., Zhang, L., Yuen, C.: Low-complexity portable passive drone surveillance via SDR-based signal processing. IEEE Commun. Mag. 56(4), 112-118 (2018)

18. Gavriluţ, V., Zhao, L., Raagaard, M.L., Pop, P.: AVB-aware routing and scheduling of time-triggered traffic for TSN. IEEE Access 6, 75229-75243 (2018)

19. Geirhofer, S., Tong, L., Sadler, B.M.: Cognitive radios for dynamic spectrum access-dynamic spectrum access in the time domain: Modeling and exploiting white space. IEEE Commun. Mag. 45(5), 66-72 (2007)

20. Goldsmith, A.J., Jafar, S.A., Maric, I., Srinivasa, S.: Breaking spectrum gridlock with cognitive radios: An information theoretic perspective. Proc. IEEE 97(5), 894-914 (2009)

21. Gomez-Ojeda, R., Gonzalez-Jimenez, J.: Robust stereo visual odometry through a probabilistic combination of points and line 
segments. In: 2016 IEEE International Conference on Robotics and Automation (ICRA). IEEE, pp 2521-2526 (2016)

22. Gu, M., Zhan, P., He, X., Yan, D.: Integration design of ipv6 and time-triggered ethernet on spacecraft. In: International Conference on Wireless and Satellite Systems. Springer, pp 392-400 (2019)

23. Gupta, S.G., Ghonge, M.M., Jawandhiya, P.: Review of unmanned aircraft system (UAS). Int. J. Adv. Res. Comput. Eng. Technol. (IJARCET) 2(4), 1646-1658 (2013)

24. Habibi, M.A., Nasimi, M., Han, B., Schotten, H.D.: A comprehensive survey of ran architectures toward $5 \mathrm{~g}$ mobile communication system. IEEE Access 7, 70371-70421 (2019)

25. Hawilo, H., Jammal, M., Shami, A.: Network function virtualization-aware orchestrator for service function chaining placement in the cloud. IEEE J. Sel. Areas Commun. 37(3), 643-655 (2019). https://doi.org/10.1109/JSAC.2019.2895226

26. Hawilo, H., Shami, A., Mirahmadi, M., Asal, R.: Nfv: state of the art, challenges, and implementation in next generation mobile networks (vepc). IEEE Netw. 28(6), 18-26 (2014)

27. Hayat, S., Yanmaz, E., Muzaffar, R.: Survey on unmanned aerial vehicle networks for civil applications: A communications viewpoint. IEEE Commun. Surv. Tutorials 18(4), 2624-2661 (2016)

28. Haykin, S.: Cognitive radio: brain-empowered wireless communications. IEEE J. Sel. Areas Commun. 23(2), 201-220 (2005)

29. Huang, J., Berry, R.A., Honig, M.L.: Auction-based spectrum sharing. Mob. Netw. Appl. 11(3), 405-408 (2006)

30. Jacob, P., Sirigina, R.P., Madhukumar, A., Prasad, V.A.: Cognitive radio for aeronautical communications: A survey. IEEE Access 4, 3417-3443 (2016)

31. Jain, R., Templin, F.: Requirements, challenges and analysis of alternatives for wireless datalinks for unmanned aircraft systems. IEEE J. Sel. Areas Commun. 30(5), 852-860 (2012). https://doi.org/10.1109/JSAC.2012.120602

32. Jeong, S., Simeone, O., Kang, J.: Mobile edge computing via a uav-mounted cloudlet: Optimization of bit allocation and path planning. IEEE Trans. Veh. Technol. 67(3), 2049-2063 (2018)

33. Jiang, T., Geller, J., Ni, D., Collura, J.: Unmanned aircraft system traffic management: Concept of operation and system architecture. Int. J. Transp. Sci. Technol. 5(3), 123-135 (2016)

34. Sun, K., Jianbin, Q., Karimi, H.R., Fu, Y.: Event-triggered robust fuzzy adaptive finite-time control of nonlinear systems with prescribed performance. IEEE Trans. Fuzzy Syst., pp 1-1. https://doi.org/10.1109/TFUZZ.2020.2979129 (2020)

35. Kassar, M., Kervella, B., Pujolle, G.: An overview of vertical handover decision strategies in heterogeneous wireless networks. Comput. Commun. 31(10), 2607-2620 (2008)

36. Ke, D., Huang, Z., Wang, X., Li, X.: Blind detection techniques for non-cooperative communication signals based on deep learning. IEEE Access 7, 89218-89225 (2019)

37. Khawaja, W., Guvenc, I., Matolak, D.W., Fiebig, U.C., Schneckenberger, N.: A survey of air-to-ground propagation channel modeling for unmanned aerial vehicles. IEEE Commun. Surv. Tutorials 21(3), 2361-2391 (2019)

38. Kitindi, E.J., Fu, S., Jia, Y., Kabir, A., Wang, Y.: Wireless network virtualization with sdn and c-ran for $5 \mathrm{~g}$ networks: Requirements, opportunities, and challenges. IEEE Access 5, 19099-19115 (2017)

39. Kliks, A., Kryszkiewicz, P., Umbert, A., Pérez-Romero, J., Casadevall, F., Kulacz, Ł.: Application of radio environment maps for dynamic broadband access in tv bands in urban areas. IEEE Access 5, 19842-19863 (2017)
40. Kumar, K., Prakash, A., Tripathi, R.: Context aware spectrum handoff scheme in cognitive radio vehicular networks. Int. J. Ad Hoc Ubiquit. Comput. 24(1-2), 101-116 (2017)

41. Li, Z., Wan, H., Pang, Z., Chen, Q., Deng, Y., Zhao, X., Gao, Y., Song, X., Gu, M.: An enhanced reconfiguration for deterministic transmission in time-triggered networks. IEEE/ACM Trans. Netw. 27(3), 1124-1137 (2019). https://doi.org/10.1109/TNET.2019. 2911272

42. Liu, H., Wei, Z., Chen, Y., Pan, J., Lin, L., Ren, Y.: Drone detection based on an audio-assisted camera array. In: 2017 IEEE Third International Conference on Multimedia Big Data (BigMM), pp 402-406 (2017)

43. Lu, Y., Sun, B.: The fitness evaluation model of saas for enterprise information system. In: 2009 IEEE International Conference on e-Business Engineering. IEEE, pp 507-511 (2009)

44. Luck, R., Ray, A.: Experimental verification of a delay compensation algorithm for integrated communication and control systems. Int. J. Control. 59(6), 1357-1372 (1994)

45. Luo, S., Xiao, Y., Lin, R., Xie, X., Bi, G., Zhao, Y., Huang, J.: Opportunistic spectrum access for UAV communications towards ultra dense networks. IEEE Access 7, 175021-175032 (2019)

46. Mahmoud, S.Y.M., Mohamed, N.: Toward a cloud platform for uav resources and services. In: 2015 IEEE Fourth Symposium on Network Cloud Computing and Applications (NCCA), pp 23-30 (2015)

47. Meghanathan, N.: Review of access control models for cloud computing. Comput. Sci. Inf. Sci. 3(1), 77-85 (2013)

48. Mei, H., Wang, K., Yang, K.: Multi-layer cloud-ran with cooperative resource allocations for low-latency computing and communication services. IEEE Access 5, 19023-19032 (2017)

49. Milani, A.S., Navimipour, N.J.: Load balancing mechanisms and techniques in the cloud environments: Systematic literature review and future trends. J. Netw. Comput. Appl. 71, 86-98 (2016)

50. Mitola, J., Maguire, G.Q.: Cognitive radio: making software radios more personal. IEEE Pers. Commun. 6(4), 13-18 (1999)

51. Mohamed, N., Al-Jaroodi, J., Jawhar, I., Noura, H., Mahmoud, S.: Uavfog: A uav-based fog computing for internet of things. In: 2017 IEEE SmartWorld, Ubiquitous Intelligence Computing, Advanced Trusted Computed, Scalable Computing Communications, Cloud Big Data Computing, Internet of People and Smart City Innovation (SmartWorld/SCALCOM/UIC/ATC/CBDCom/IOP/SCI), pp 1-8 (2017)

52. Nogales, B., Sanchez-Aguero, V., Vidal, I., Valera, F., GarciaReinoso, J.: A nfv system to support configurable and automated multi-uav service deployments. In: Proceedings of the 4th ACM Workshop on Micro Aerial Vehicle Networks, Systems, and Applications, pp 39-44 (2018)

53. Peng, H., Ye, Q., Shen, X.S.: Sdn-based resource management for autonomous vehicular networks: A multi-access edge computing approach. IEEE Wirel. Commun. 26(4), 156-162 (2019)

54. Peng, M., Zhang, K., Jiang, J., Wang, J., Wang, W.: Energyefficient resource assignment and power allocation in heterogeneous cloud radio access networks. IEEE Trans. Veh. Technol. 64(11), 5275-5287 (2014)

55. Pesko, M., Javornik, T., Kosir, A., Štular, M., Mohorcic, M.: Radio environment maps: The survey of construction methods. KSII Transactions on Internet and Information Systems 8(11), 3789-3809 (2014). https://doi.org/10.3837/tiis.2014.11.008

56. Pillai, P.S., Rao, S.: Resource allocation in cloud computing using the uncertainty principle of game theory. IEEE Syst. J. 10(2), 637-648 (2016) 
57. Plass, S.: Seamless aeronautical networking through integration of data links, radios, and antennas. In: 2013 Integrated Communications, Navigation and Surveillance Conference (ICNS), pp 1-31 (2013). https://doi.org/10.1109/ICNSurv.2013.6548615

58. Rajendran, S., Calvo-Palomino, R., Fuchs, M., Van den Bergh, B., Cordobes, H., Giustiniano, D., Pollin, S., Lenders, V.: Electrosense: Open and big spectrum data. IEEE Commun. Mag. 56(1), 210-217 (2018)

59. Reyes, H., Gellerman, N., Kaabouch, N.: A cognitive radio system for improving the reliability and security of uas/uav networks. In: 2015 IEEE Aerospace Conference. IEEE, pp 1-9 (2015)

60. Reyes, H., Kaabouch, N.: Improving the reliability of unmanned aircraft system wireless communications through cognitive radio technology. Commun. Netw. 5(03), 225 (2013)

61. Rodrigues, E.M.G., Godina, R., Pouresmaeil, E., Catalão, J.P.S.: Hybrid time triggered protocol for home wireless communications. In: 2017 IEEE International Conference on Environment and Electrical Engineering and 2017 IEEE Industrial and Commercial Power Systems Europe (EEEIC / I CPS Europe), pp 1-6 (2017)

62. Saleem, Y., Rehmani, M.H., Zeadally, S.: Integration of cognitive radio technology with unmanned aerial vehicles: issues, opportunities, and future research challenges. J. Netw. Comput. Appl. 50, 15-31 (2015)

63. Sara, M., Jawhar, I., Nader, M.: A softwarization architecture for uavs and wsns as part of the cloud environment. In: 2016 IEEE International Conference on Cloud Engineering Workshop (IC2EW). IEEE, pp 13-18 (2016)

64. Sato, K., Fujii, T.: Kriging-based interference power constraint: Integrated design of the radio environment map and transmission power. IEEE Trans. Cogn. Commun. Netw. 3(1), 13-25 (2017)

65. Shakeri, R., Al-Garadi, M.A., Badawy, A., Mohamed, A., Khattab, T., Al-Ali, A., Harras, K.A., Guizani, M.: Design challenges of multi-UAV systems in cyber-physical applications: A comprehensive survey, and future directions. IEEE Commun. Surv. Tutorials 21(4), 3340-338 (2019)

66. Shamsoshoara, A., Afghah, F., Razi, A., Mousavi, S., Ashdown, J., Turk, K.: An autonomous spectrum management scheme for unmanned aerial vehicle networks in disaster relief operations. IEEE Access 8, 58064-58079 (2020)

67. Si, P., Yu, F.R., Yang, R., Zhang, Y.: Navigation dataassisted opportunistic spectrum scheduling for network-based uav systems: A parallel restless bandits formulation. Wirel. Pers. Commun. 85(1), 29-48 (2015)

68. Singh, A., Chatterjee, K.: Cloud security issues and challenges: A survey. J. Netw. Comput. Appl. 79, 88-115 (2017)

69. Sklivanitis, G., Gannon, A., Tountas, K., Pados, D.A., Batalama, S.N., Reichhart, S., Medley, M., Thawdar, N., Lee, U., Matyjas, J.D., Pudlewski, S., Drozd, A., Amanna, A., Latus, F., Goldsmith, Z., Diaz, D.: Airborne cognitive networking: Design, development, and deployment. IEEE Access 6, 47217-47239 (2018). https://doi.org/10.1109/ACCESS.2018.2857843

70. Sodagari, S.: A secure radio environment map database to share spectrum. IEEE J. Sel. Top. Sig. Process. 9(7), 1298-1305 (2015)

71. Stamatescu, G., Popescu, D., Dobrescu, R.: Cognitive radio as solution for ground-aerial surveillance through WSN and UAV infrastructure. In: Proceedings of the 2014 6th International Conference on Electronics, Computers and Artificial Intelligence (ECAI). IEEE, pp 51-56 (2014)
72. Stankovic, J.A., Abdelzaher, T.E., Chenyang, L.u., Lui S.: Real-time communication and coordination in embedded sensor networks. Proc. IEEE 91(7), 1002-1022 (2003)

73. Sulieman, N.I., Balevi, E., Gitlin, R.D.: Near-instant link failure recovery in $5 \mathrm{~g}$ wireless fog-based-fronthaul networks. In: 2018 Wireless Telecommunications Symposium (WTS). IEEE, pp. 1-6 (2018)

74. Sun, K., Liu, L., Qiu, J., Feng, G.: Fuzzy adaptive finite-time fault-tolerant control for strict-feedback nonlinear systems. IEEE Trans. Fuzzy Syst., pp 1-1. https://doi.org/10.1109/TFUZZ.2020. 2965890 (2020)

75. Sun, K., Qiu, J., Karimi, H.R., Gao, H.: A novel finite-time control for nonstrict feedback saturated nonlinear systems with tracking error constraint. IEEE Trans. Systems Man Cybern. Syst., pp 1-12. https://doi.org/10.1109/TSMC.2019.2958072 (2019)

76. Tipantuña, C., Hesselbach, X., Sánchez-Aguero, V., Valera, F., Vidal, I., Nogales, B.: An NFV-based energy scheduling algorithm for a $5 \mathrm{G}$ enabled fleet of programmable unmanned aerial vehicles. Wireless Communications and Mobile Computing 2019, 1-20 (2019)

77. Tsiropoulos, G.I., Dobre, O.A., Ahmed, M.H., Baddour, K.E.: Radio resource allocation techniques for efficient spectrum access in cognitive radio networks. IEEE Commun. Surv. Tutorials 18(1), 824-847 (2014)

78. Wang, L.C., Wang, C.W.: Spectrum handoff for cognitive radio networks: Reactive-sensing or proactive-sensins? In: 2008 IEEE International Performance, Computing and Communications Conference. IEEE, pp 343-348 (2008)

79. White, K.J., Denney, E., Knudson, M.D., Mamerides, A.K., Pezaros, D.P.: A programmable SDN+ NFV-based architecture for UAV telemetry monitoring. In: 2017 14th IEEE Annual Consumer Communications \& Networking Conference (CCNC). IEEE, pp 522-527 (2017)

80. Wong, A.H., Neve, M.J., Sowerby, K.W.: Antenna selection and deployment strategies for indoor wireless communication systems. IET Commun. 1(4), 732-738 (2007)

81. Wu, L., Garg, S.K., Versteeg, S., Buyya, R.: Sla-based resource provisioning for hosted software-as-a-service applications in cloud computing environments. IEEE Trans. Serv. Comput. 7(3), $465-485$ (2014)

82. Xu, X., Dou, W., Zhang, X., Chen, J.: Enreal: An energy-aware resource allocation method for scientific workflow executions in cloud environment. IEEE Trans. Cloud Comput. 4(2), 166-179 (2016)

83. Xu, Z., Petrunin, I., Tsourdos, A., Sabyasachi, M., Williamson, A.: Cognitive communication scheme for unmanned aerial vehicle operation. In: 2019 Workshop on Research, Education and Development of Unmanned Aerial Systems (RED UAS). IEEE, pp 271-277 (2019)

84. Yan, X., Seekercioğlu, Y.A., Narayanan, S.: A survey of vertical handover decision algorithms in fourth generation heterogeneous wireless networks. Comput. Netw. 54(11), 1848-1863 (2010)

85. Younis, Y.A., Kifayat, K., Merabti, M.: An access control model for cloud computing. J. Inf. Secur. Appl. 19(1), 45-60 (2014)

86. Yuan, Y., Sun, Z., Wei, Z., Jia, K.: Deepmorse: A deep convolutional learning method for blind morse signal detection in wideband wireless spectrum. IEEE Access 7, 80577-80587 (2019)

87. Sun, Y., Xiao, Z., Bao, D., Zhao, J.: An architecture model of management and monitoring on cloud services resources. In: 2010 3rd International Conference on Advanced Computer Theory and Engineering(ICACTE), vol. 3, pp V3-207-V3-211 (2010) 
88. Zeng, Y., Liang, Y.C., Hoang, A.T., Zhang, R.: A review on spectrum sensing for cognitive radio: challenges and solutions. EURASIP J. Adv. Sig. Process. 2010, 2 (2010)

89. Zhan, Z.H., Liu, X.F., Gong, Y.J., Zhang, J., Chung, H.S.H., $\mathrm{Li}$, Y.: Cloud computing resource scheduling and a survey of its evolutionary approaches. ACM Comput. Surv. (CSUR) 47(4), 1-33 (2015)

90. Zhang, S., Zeng, Y., Zhang, R.: Cellular-enabled UAV communication: A connectivity-constrained trajectory optimization perspective. IEEE Trans. Commun. 67(3), 2580-2604 (2018)

91. Zhang, Y., He, F., Lu, G., Xiong, H.: Scheduling rate-constrained flows with dynamic programming priority in time-triggered ethernet. Chin. J. Electron. 26(4), 849-855 (2017)

92. Zhao, L., Xiong, H., Zheng, Z., Li, Q.: Improving worst-case latency analysis for rate-constrained traffic in the time-triggered ethernet network. IEEE Commun. Lett. 18(11), 1927-1930 (2014)

93. Zheng, H., Cao, L.: Device-centric spectrum management. In: First IEEE International Symposium on New Frontiers in Dynamic Spectrum Access Networks, 2005. DySPAN 2005. IEEE, pp 56-65 (2005)

94. Zhidong, L., Xiaolin, Z.: A novel frequency hopping scheme based on cognitive radio for aircraft. In: 2013 IEEE International Conference on Green Computing and Communications and IEEE Internet of Things and IEEE Cyber, Physical and Social Computing, pp 1231-1236 (2013)

Publisher's Note Springer Nature remains neutral with regard to jurisdictional claims in published maps and institutional affiliations.

Zhengjia Xu received his B.S. degree in Civil Aviation Electrical and Electronic Engineering, and his M.S. degree in Vehicle Operation Engineering from Nanjing University of Aeronautics and Astronautics, Nanjing, China, in 2014 and 2017, separately. He is currently working toward his Ph.D. degree at Cranfield University. His current research interests are: cognitive communication for $\mathrm{UAV}_{\mathrm{s}}$, spectrum allocation and management, spectrum identification, and the integrated system design.
Ivan Petrunin received his MSc degree in Design and Manufacturing of Electronic Equipment from National Technical University of Ukraine in 1998, PhD degree in Signal Processing for Condition Monitoring from Cranfield University, UK, in 2013. At the present time he holds position of Lecturer in Digital Signal Processing in the Centre for Autonomous and Cyber-Physical Systems at Cranfield University. His current research focus is in areas of applied signal processing for autonomous systems with particular emphasis on communication, navigation and surveillance aspects, such as cognitive communication schemes, assured multi-sensor navigation and remote sensing.

Antonios Tsourdos received the M.Eng. degree in electronic, control and systems engineering from the University of Sheffield, Sheffield, U.K., in 1995, the M.Sc. degree in systems engineering from Cardiff University, Cardiff, U.K., in 1996, and the Ph.D. degree in nonlinear robust missile autopilot design and analysis from Cranfield University, Cranfield, U.K. in 1999. He is currently a Professor of control systems with Cranfield University, where he was the Appointed Head of the Autonomous Systems Group in 2007. He has published over 100 peer-reviewed journal and conference papers. Prof. Tsourdos was a member of the Team Stellar, the winning team for the U.K. MoD Grand Challenge in 2008 and the IET Innovation Award (Category Team, 2009). He is an editorial board member on several international publications, including IMechE and IEEE. He is a member of the IFAC Technical Committee on Intelligent Autonomous Vehicles, the IET Executive Team on Robotics and Automation, and the ATI Autonomous Systems National Technical Committee. He was also involved in the SEAS DTC on Autonomous Systems Verifications. He has also been engaged in research on guidance and control for single and multiple vehicles as well as verifiable autonomy of autonomous systems and lately dealing with the newly important subjects of integrated system health management and cyber-physical systems. 
2021-02-03

\title{
Dynamic spectrum management with network function virtualization for UAV communica
}

\author{
Xu, Zhengjia
}

Springer

Xu Z, Petrunin I, Tsourdos A. (2021) Dynamic spectrum management with network function virtualization for UAV communication. Journal of Intelligent and Robotic Systems, Volume 101, February 2021, Article number 40 https://doi.org/10.1007/s10846-021-01318-0

Downloaded from Cranfield Library Services E-Repository 